\title{
Intranodal lymphangiography for chyle leakage after esophagectomy: A case report
}

\author{
TOMONARI SUETSUGU ${ }^{1}$, YOSHIHIRO TANAKA ${ }^{1}$, SHINYA BANNO $^{1}$, MASAHIRO FUKADA ${ }^{1}$, \\ ITARU YASUFUKU $^{1}$, YOSHINORI IWATA ${ }^{1}$, TAKEHARU IMAI ${ }^{1}$, TOSHIYUKI TANAHASHI $^{1}$, SATOSHI MATSUI $^{1}$, \\ HISASHI IMAI $^{1}$, NOBUHISA MATSUHASHI ${ }^{1}$, TAKAO TAKAHASHI ${ }^{1}$, KAZUYA YAMAGUCHI $^{1}$, \\ YUKICHI TANAHASHI ${ }^{2}$, HIROSHI KAWADA ${ }^{2}$, MASAYUKI MATSUO ${ }^{2}$ and KAZUHIRO YOSHIDA ${ }^{1}$
}

Departments of ${ }^{1}$ Surgical Oncology and ${ }^{2}$ Radiology,

Gifu University School of Medicine, Gifu 501-1194, Japan

Received April 27, 2019; Accepted January 15, 2020

DOI: $10.3892 /$ mco.2020.1990

\begin{abstract}
Postoperative chylothorax after esophagectomy is a relatively rare complication, but treatment can sometimes be complicated. We report 3 cases of Lipiodol lymphangiography via inguinal lymph node puncture that was effective for chyle leakage occurring after esophagectomy. Case 1: A 67-year-old man with stage IIIA esophageal squamous cell carcinoma underwent radical esophagectomy by video-assisted thoracic surgery (VATS) following neoadjuvant chemotherapy (NAC). After enteral feeding, right pleural effusion drainage increased sharply and changed to white color that was diagnosed as chylothorax. Conservative treatment was started on postoperative day (POD) 15. On POD 50, intranodal Lipiodol lymphangiography and thoracic duct ligation were performed, resulting in complete improvement by the next day. Case 2: A 69-year-old man with stage IIIC esophageal cancer was treated salvage operation following chemoradiation. Postoperative chylothorax was diagnosed on POD 6. Despite conservative treatment, the pleural fluid volume did not decrease. Intranodal Lipiodol lymphangiography performed on POD 13 showed contrast medium draining from the thoracic duct near the tracheal bifurcation. Thoracotomy for thoracic duct ligation was performed on POD 15. Thereafter, drainage from the thoracic drain decreased significantly, and the right thoracic drain was removed 4 days later. Case 3: A 65-year-old man with Stage IVA hypopharyngeal cancer and Stage IIIA esophageal cancer underwent total pharyngopharyngeal
\end{abstract}

Correspondence to: Professor Kazuhiro Yoshida, Department of Surgical Oncology, Gifu University School of Medicine, 1-1 Yanagido, Gifu 501-1194, Japan

E-mail:kyoshida@gifu-u.ac.jp

Abbreviations: VATS, video-assisted thoracic surgery; Bi-DCF, biweekly docetaxel/cisplatin/5-FU chemotherapy; NAC, neoadjuvant chemotherapy; POD, postoperative day

Key words: chylothorax, esophagectomy, lymphangiography esophagectomy by VATS following NAC. Postoperative chylothorax was diagnosed on POD 7. Despite conservative treatment, the pleural fluid volume did not decrease. Intranodal Lipiodol lymphangiography performed on POD 19 completely visualized the thoracic duct and showed no outflow of contrast from the main thoracic duct into the mediastinum. Pleural fluid decreased remarkably after lymphangiography. Intranodal Lipiodol lymphangiography for postoperative chylothorax accurately visualizes flow within the thoracic duct and clearly depicts its positional relationship with other organs. Besides lymphangiography is not only helps to determine the site of chyle leakage but can also be effective for curing chylothorax by less invasive and safer method.

\section{Introduction}

Postoperative chylothorax after esophagectomy is a relatively rare complication with a reported incidence rate ranging from 1.1 to $3.9 \%$ (1). However, once it occurs, chylothorax can lead to serious complications such as significant impairment of the cardiovascular and respiratory systems, nutritional status, and immune system unless appropriately treated $(2,3)$. Treatment of chylothorax includes conservative treatment and surgical treatment. Conservative treatment including total parenteral nutrition, no enteral intake, and octreotide is initially chosen, but if the chylothorax is not improved, surgical treatment is often required (4). However surgical treatment may be highly invasive for patients with postoperative chylothorax who are frequently in a poor nutritional and immunosuppressive condition, and lymphangiography must be considered before surgical treatment. Although the traditional lymphangiography procedure uses a bilateral pedal approach (5), recent studies have suggested the use of ultrasound-guided intranodal lymphangiography because of its simplicity and safety $(6,7)$. Intranodal lymphangiography involving inguinal lymph node puncture is a feasible and useful treatment in terms of not only the way in which it helps determine the site of chyle leakage but also as an effective therapeutic modality for treating chylothorax.

The purpose of this study is to clarify the efficacy of lymphangiography for chyle leakage. We herein report three 

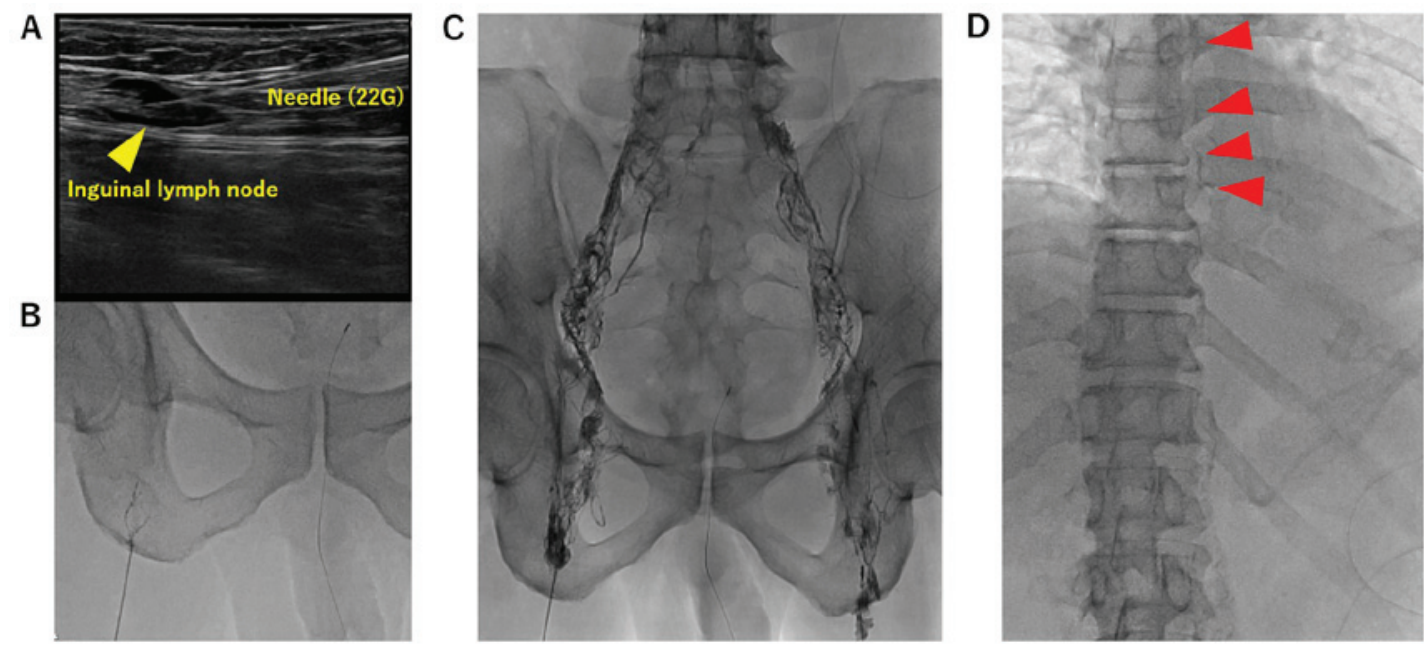

Figure 1. Representative images of the procedure in case 1. (A) Puncture of both sides of the inguinal lymph nodes (yellow arrowhead). (B) Injection of Lipiodol (1 ml over $3 \mathrm{~min})$ and detection of the lymphatic duct. (C) Contrast injection to visualize the lymph nodes around the common iliac vessels and the para-aortic lymph nodes. (D) Visualization of the thoracic duct (red arrowheads).

cases of esophagectomy complicated by chyle leakage. In two of them, lymphangiography visualized the movement of the thoracic duct and clearly depicted its positional relationship with other organs which lead to appropriate surgical treatment. In one case, chyle leakage was successfully treated by lymphangiography as embolization of micro-injury in thoracic duct.

\section{Case report}

Case 1. A 67-year-old man was diagnosed as having middle thoracic esophageal squamous cell carcinoma of cT3N1M0 cStage IIIA (UICC 8th edition). He underwent radical esophagectomy by video-assisted thoracic surgery (VATS) following 2 cycles of neoadjuvant biweekly-DCF chemotherapy (docetaxel $35 \mathrm{mg} / \mathrm{m}^{2}$ on days 1 and 15 ; cisplatin $40 \mathrm{mg} / \mathrm{m}^{2}$ on days 1 and 15; and 5-FU $400 \mathrm{mg} / \mathrm{m}^{2} /$ day on days $1-5$ and $15-19$; Bi-DCF). Operation time was $515 \mathrm{~min}$, blood loss was $145 \mathrm{~g}$, and the thoracic duct was preserved. After enteral feeding was started on postoperative day (POD) 2, right pleural effusion drainage increased to $1,360 \mathrm{ml}$ daily and changed to a milky white color. The patient then was diagnosed as having postoperative chylothorax. After conservative treatment including fat restriction was started on the same day, the chylothorax improved for a few days. However, he complained of breathing difficulties after enteral nutrition that included added fat. Re-exacerbation of the chylothorax up to $1,500 \mathrm{ml}$ daily was observed on POD 15, and conservative treatment was started once again. On POD 50, ultrasound-guided intranodal Lipiodol lymphangiography involving inguinal lymph node puncture (Fig. 1) was performed that revealed Lipiodol leakage $4 \mathrm{~cm}$ on the caudal side of the tracheal bifurcation along the thoracic duct (Fig. 2A). Ligation of the thoracic duct by VATS was performed following the lymphangiography. Thoracic duct was identified in the lower mediastinum, and clipping was performed on the caudal side of the injured part (Fig. 2A). The patient's chylothorax improved completely by the next day, and the right thoracic drain was removed 7 days after the operation (Fig. 2C).
Case 2. A 69-year-old man was diagnosed as having lower esophageal squamous cell carcinoma of cT3N3M0 cStage IIIC (UICC 8th edition). He was initially started on chemoradiotherapy (radiation 2 Gy for 30 days and 2 cycles of Bi-DCF). Although remarkable tumor shrinkage was obtained initially, the tumor relapsed 5 months later. Therefore, esophagectomy was performed as a salvage operation. Operation time was $367 \mathrm{~min}$, blood loss was $260 \mathrm{~g}$, and the thoracic duct was preserved. After enteral feeding was started on POD 3, right pleural effusion drainage increased to $3,790 \mathrm{ml}$ on POD 6 and chylothorax was diagnosed. The patient was started on conservative treatment, but the volume of pleural fluid did not decrease. Intranodal Lipiodol lymphangiography was performed on POD 13, and contrast medium was observed draining from the thoracic duct near the tracheal bifurcation (Fig. 3A). We performed a thoracotomy on POD 15 for ligation of the thoracic duct. Thereafter, the volume of drainage from the thoracic drain decreased significantly, and the right thoracic drain was removed 5 days after the operation (Fig. 3B).

Case 3. A 65-year-old man was diagnosed as having hypopharyngeal squamous cell carcinoma of cT4aN0M0 cStage IVA (AJCC Cancer Staging Manual 8th edition) and upper thoracic esophageal squamous cell carcinoma of cT3N1M0 cStage IIIA (UICC 8th edition). He underwent total pharyngopharyngeal esophagectomy by VATS following 1 cycle of neoadjuvant chemotherapy (NAC) (cisplatin $80 \mathrm{mg} / \mathrm{m}^{2}$ on days 1 and $5-\mathrm{FU}$ $800 \mathrm{mg} / \mathrm{m}^{2}$ on days $1-5$ ). Operation time was $755 \mathrm{~min}$, blood loss was $620 \mathrm{~g}$, and the thoracic duct was preserved. Enteral feeding was started on POD 4, but ascites fluid appeared from the upper wound edge on POD 5. Besides, the pleural fluid increased to $3,140 \mathrm{ml}$ and turned white color on POD7. He was diagnosed as having postoperative chylothorax on the same day. Although he was started on conservative treatment, the volume of pleural fluid did not decrease. After cessation of enteral nutrition on POD 13, pleural effusion gradually decreased, but due to concerns about re-enhancing pleural effusion following resumption of nutrition, intranodal lymphangiography was performed on POD 19. The thoracic duct was completely visualized and showed small 

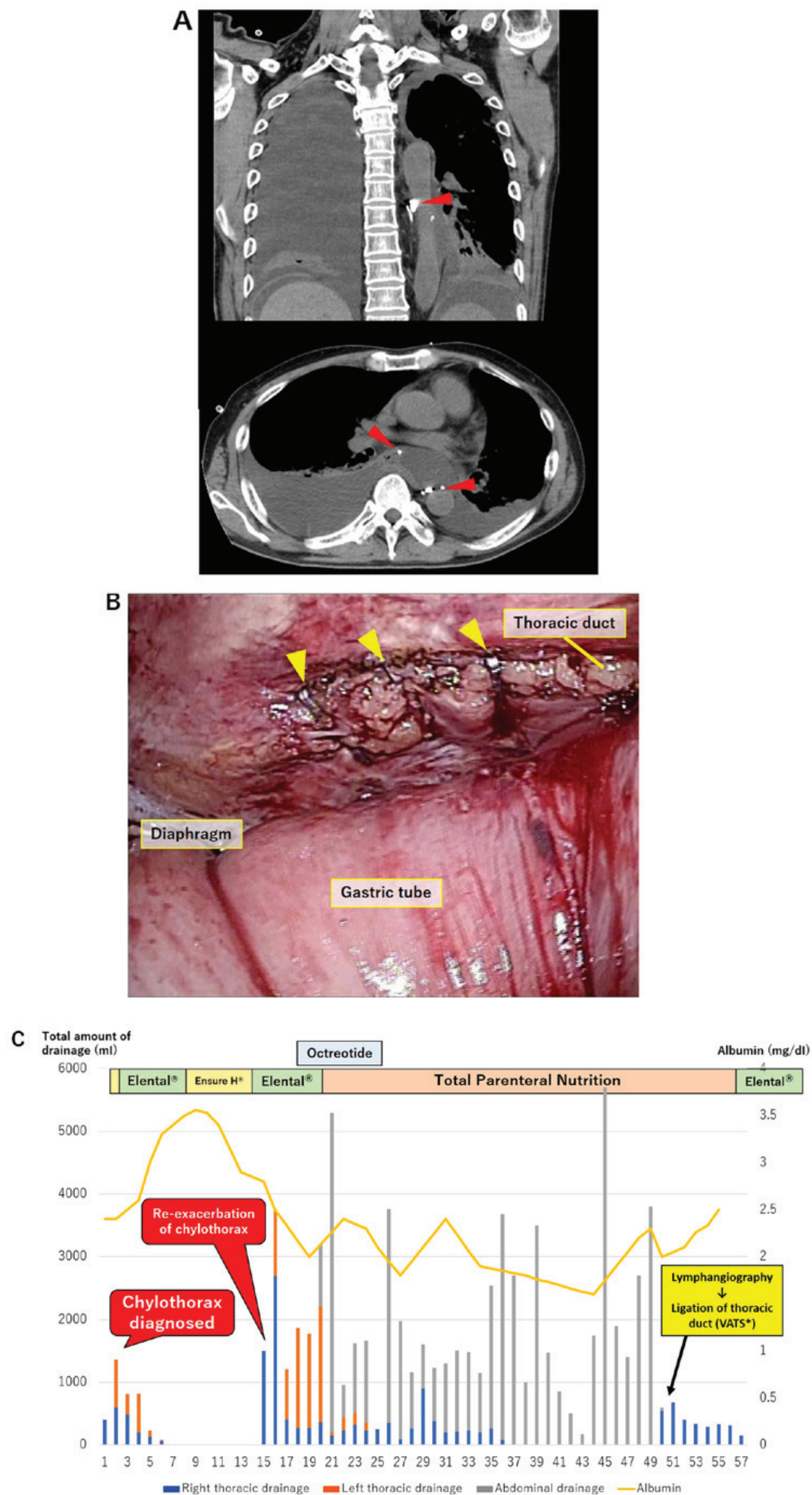

Figure 2. (A) Representative computed tomography images of case 1: A contrast effect in the proximal side of the thoracic duct could not be confirmed. Movement of contrast medium to the right thoracic cavity was noted $4 \mathrm{~cm}$ on the caudal side of the tracheal bifurcation along the thoracic duct (red arrowheads). (B) Thoracic duct ligation performed under VATS in case 1. Esophagectomy with preserving the thoracic duct and gastric tube reconstruction via the posterior mediastinum was performed in previous surgery. The thoracic duct was identified cranial from the diaphragm and ligated with three clips (yellow arrowheads). (C) Clinical course of case 1. VATS, Video Assisted Thoracic Surgery. 

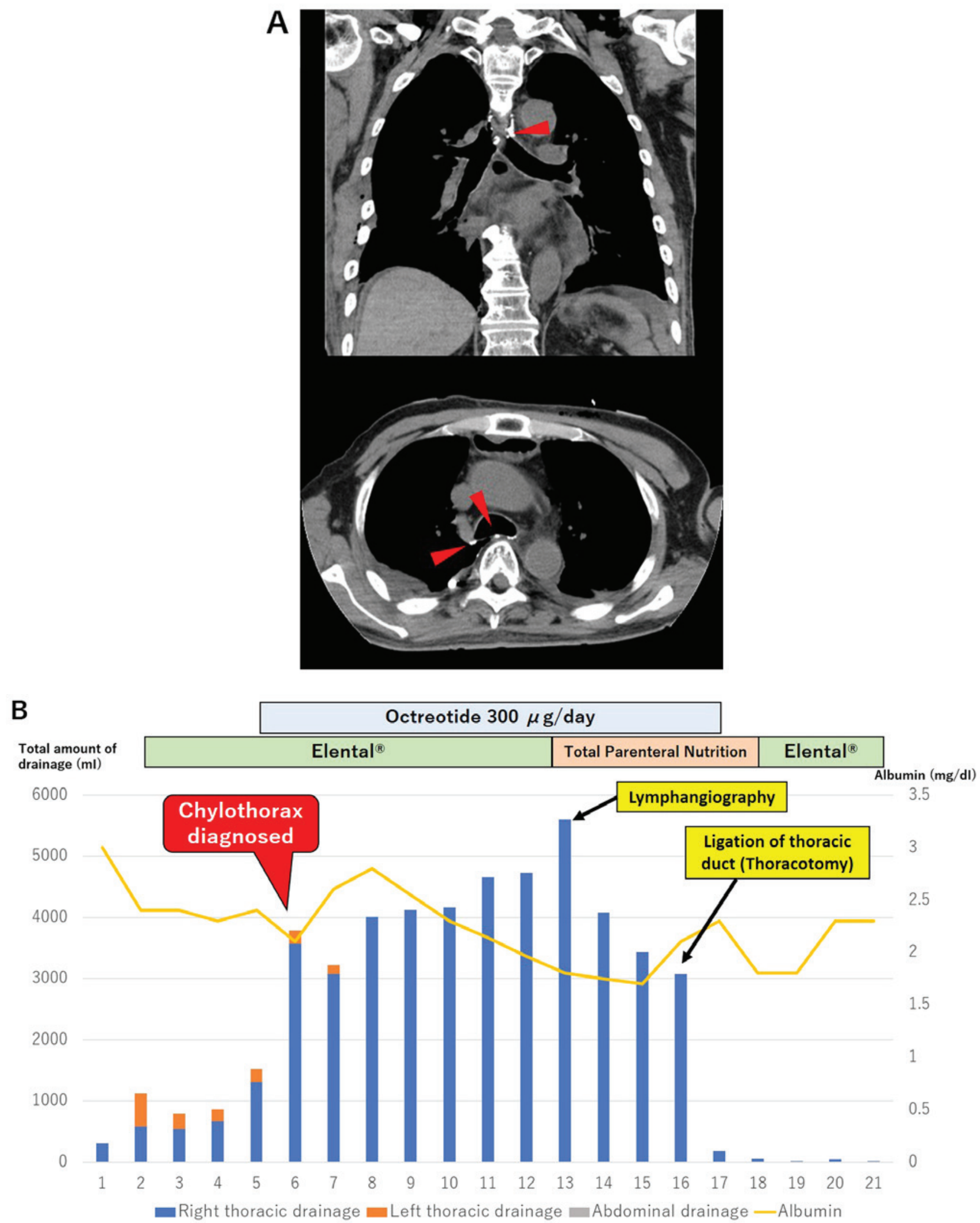

Figure 3. (A) Representative computed tomography images of case 2: Although no major injury to the main thoracic duct was obvious, outflow of contrast medium was noted at the same level as the tracheal bifurcation along the thoracic duct (red arrowheads). (B) Clinical course of case 2.

outflow of Lipiodol contrast into the mediastinum from the thoracic duct (Fig. 4A). The volume of pleural fluid decreased and there was no re-progression after lymphangiography. All drainage tubes were removed 8 days after the procedure (Fig. 4B).

\section{Discussion}

The cause of chylothorax as a postoperative complication after surgery for esophageal cancer appears to be thoracic duct 


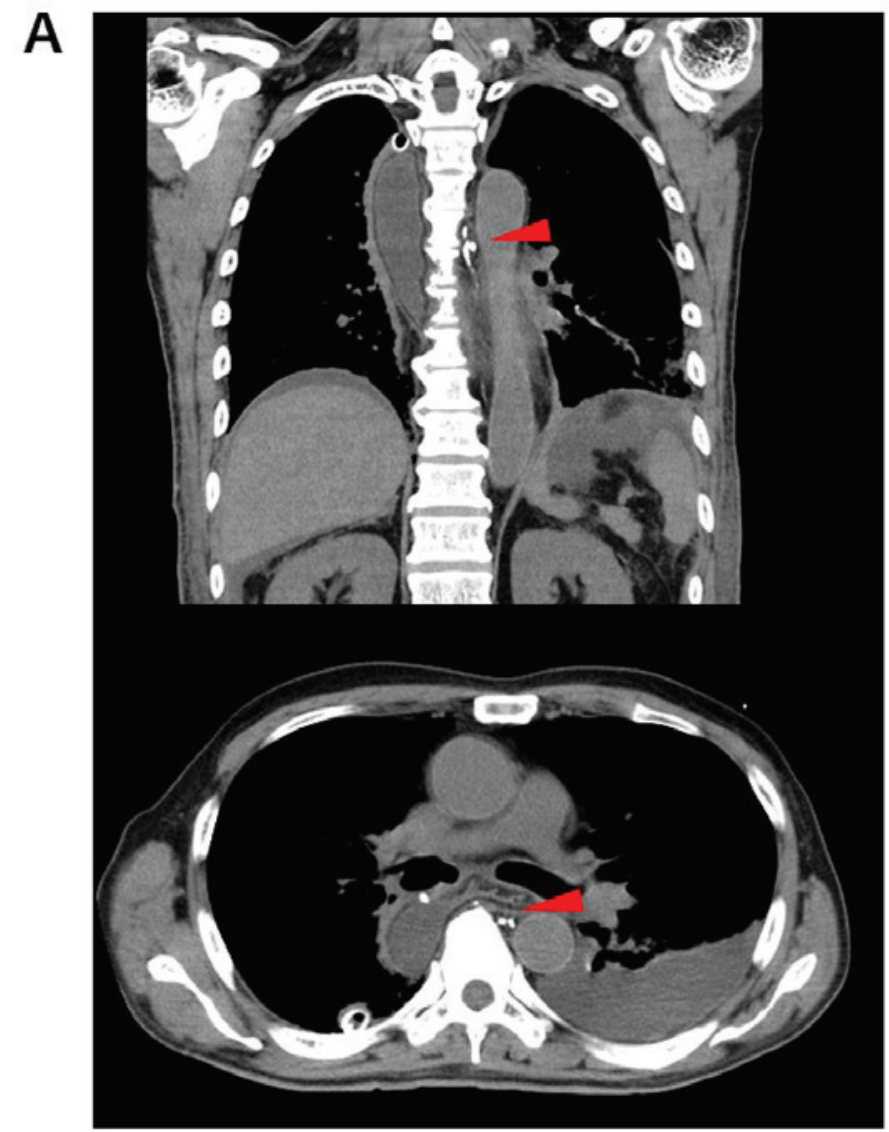

B

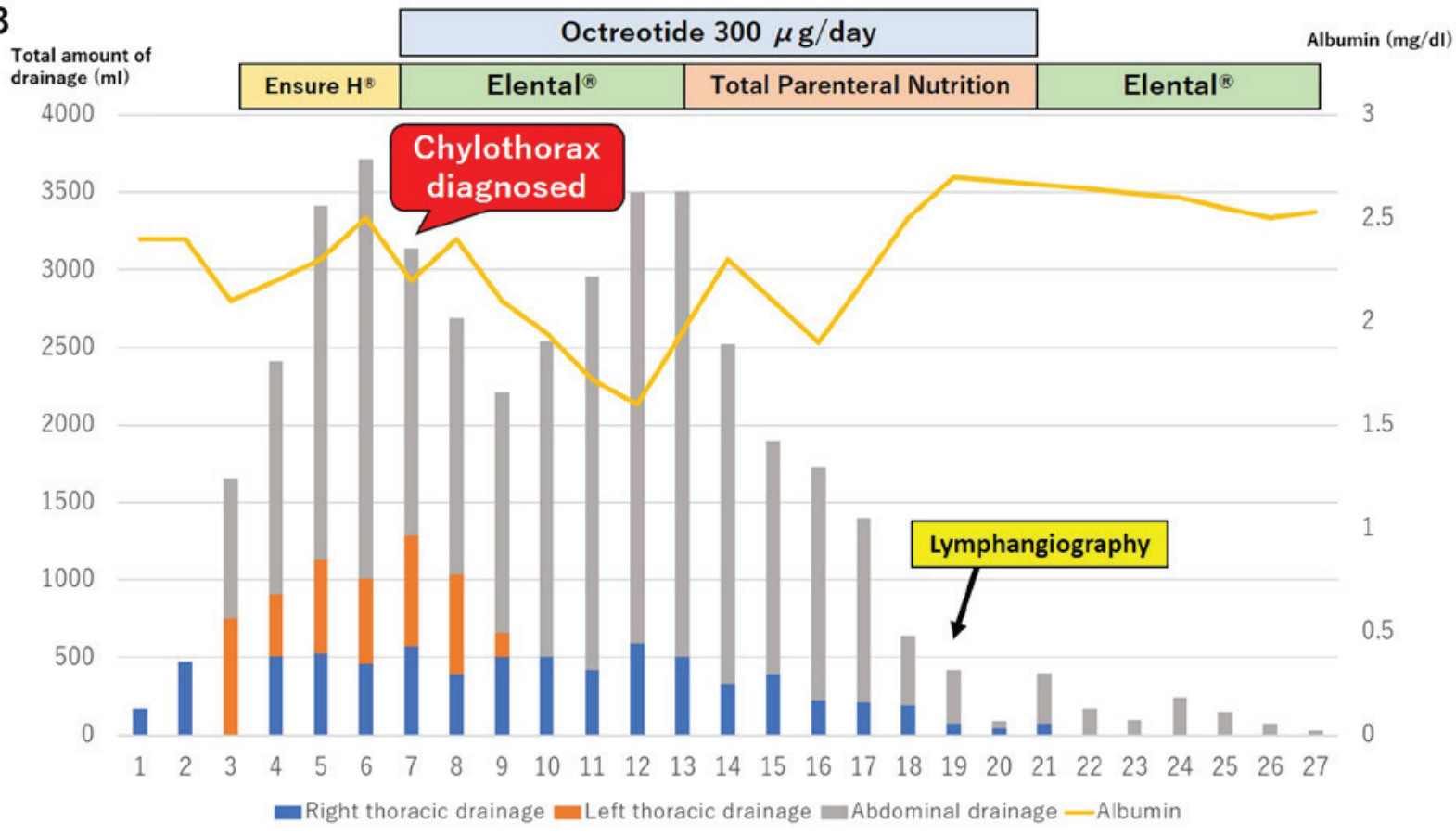

Figure 4. (A) Representative computed tomography images of case 3: The thoracic duct was completely enhanced. Leakage of contrast medium was noted $1 \mathrm{~cm}$ on the caudal side of the tracheal bifurcation along the thoracic duct (red arrowheads). (B) Clinical course of case 3 .

injury associated with the surgical intervention. Furthermore, an increased incidence of postoperative chylothorax has recently been reported most notably following increasingly radical procedures that are performed after neoadjuvant chemoradiotherapy (8).
Although the thoracic duct can have a variable course, it is usually detected at the level of the diaphragm where it passes through the aortic hiatus with the aorta and azygous vein in the posterior mediastinum. The thoracic duct then continues its course between the aorta and azygos vein and crosses to 
the left side of the body at either the fifth or sixth thoracic vertebra. The thoracic duct runs posterior to the aortic arch and next to the esophagus until it drains into the junction of the left subclavian and internal jugular veins, where lymph enters the systemic circulation (9).

Two of our three patients received preoperative chemotherapy and one received preoperative chemoradiotherapy. According to the esophageal cancer treatment guidelines in Japan, combined therapy with cisplatin and 5-FU is standard preoperative chemotherapy (10), but the effectiveness of triplet regimens has also been reported (11-13). At our institution, Bi-DCF therapy is used as a potent triple regimen for preoperative chemotherapy, and a $90.3 \%$ response rate is reported (14). There is a report of increased postoperative chylothorax following chemoradiotherapy (8), and we have also experienced one case, but it is also possible that postoperative chylothorax is likely to occur in cases in which a tumor located beside the thoracic duct shrinks significantly due to effective NAC.

An early indication of chylothorax includes a daily chest tube output of more than $400 \mathrm{ml}$. Besides, chylothorax is readily diagnosed by the detection of milky white fluid draining from the chest after the initiation of enteral nutrition. High triglyceride levels and the demonstration of Sudan III-stained fat droplets in the pleural effusion are also diagnostic factors. Triglyceride values $>110 \mathrm{mg} / \mathrm{dl}$ are highly suggestive of a chylous effusion (15).

The thoracic duct transports between 1.5 and 41 of chyle per day back into the bloodstream in a normal adult. Hypovolemia may be present due to intravascular volume depletion. Electrolyte imbalance following chylothorax can also result in metabolic acidosis, hyponatremia, or hypocalcemia. In addition, the loss of proteins, fat-soluble vitamins, lipids, and electrolytes leads to nutritional deficiency. A decrease in cellular and humoral immunity (hypogammaglobulinemia) ultimately leaves the patient immunosuppressed and susceptible to infection and sepsis (16). The greater the duration of chyle leakage, the greater are the detrimental effects, and thus the need for urgent treatment.

Initial treatment of chylothorax is usually conservative and includes fat restriction, fasting, and total parenteral nutrition to reduce the flow of lymph fluid. In addition, albumin supplementation is also examined for malnutrition due to the loss of lymph fluid.

The effectiveness of the administration of octreotide, a long-acting somatostatin analogue, was also reported in the treatment of chylothorax (17). If the patient does not show signs of improvement, lymphangiography is considered before surgical therapy.

Recently, several studies showed good outcomes following interventional radiology embolization of the thoracic duct using a vascular coil or lipid material (5). Itkin et al (5) reported that the cure rate was $71 \%$ in 109 patients who needed treatment for chylothorax due to thoracic duct injuries while safety was maintained. Besides, to reduce invasiveness and ensure safety, intranodal lymphangiography involving inguinal lymph node puncture was reported to be a feasible and useful treatment for patients $(6,7)$.

If conservative treatment fails, surgical treatment is often considered. Direct thoracic duct ligation via VATS and open surgery are popular and successful means of resolving postoperative chylothorax. However, indications and timing for surgical treatment are controversial. Some authors recommend ligation of the thoracic duct when the amount of chylous drainage exceeds $1,000 \mathrm{ml}$ for $>7$ days $(18,19)$. Miao et al $(4)$ reported medical management was more likely to fail in patients who had chest tube drainage volume of more than $13.5 \mathrm{ml} / \mathrm{kg}$ on the third day after initiation of conservative treatment.

In this study, though one patient showed improvement of his chylothorax after lymphangiography, the other two patients required surgical treatment. In case 1 , the amount of pleural effusion varied greatly, and even though it seemed to have improved once, the course that the amount of drainage increased the next day was repeated. The decision to perform lymphangiography was delayed. There was also a gradual decrease in nutrition, and the contrast medium did not stay in the damaged area when lymphangiography was performed, and the contrast medium through thoracic duct flowed out to the intrathoracic cavity. It was judged that lymphangiography alone was inadequate. Besides, this patient was originally performed esophagectomy by VATS, and thoracic duct ligation with VATS was deemed appropriate. In case 2, due to radical chemoradiotherapy and salvage operation, his capacity to receive the treatment was declining when he diagnosed chylothorax. Therefore, conservative treatment with simultaneous enteral nutrition excluding fat was considered. However, there is no improvement of chylothorax exceeding $3,000 \mathrm{ml}$ daily for a week and lymphangiography was performed. Lymphangiography showed that the contrast medium though thoracic duct flowed out to the intrathoracic cavity similar to case 1 . He was performed thoracic duct ligation in a few days. On the other hand, in case 3, lymphangiography was performed when pleural effusion was decreasing $<1,000 \mathrm{ml}$ daily. Contrast medium from the thoracic duct remained in the mediastinum around the leak point. Considering these cases, large amount of pleural effusion over 1,000 $\mathrm{ml}$ daily and the flowing out of contrast medium to intrathoracic cavity seem to be indications to required surgical treatment despite performing lymphangiography.

Lymphangiography was performed without complications in all cases. Improvement in nutritional status and serum albumin level was observed after chylothorax treatment.

In conclusion, ultrasound-guided intranodal Lipiodol lymphangiography involving inguinal lymph node puncture not only helps to determine the site of chyle leakage but can also be effective for curing chylothorax. Besides, poor improvement with conservative treatment, including lymphangiography, are good indications for surgical treatment. Safe and appropriate treatment for postoperative chylothorax following esophagectomy needs to be established.

\section{Acknowledgements}

Not applicable.

\section{Funding}

No funding was received. 


\section{Availability of data and materials}

All data generated or analyzed during this study are included in this published article.

\section{Authors' contributions}

TS and YoT contributed to study conception and design. TS, YoT, SB, MF, IY, YI, TI, ToT, SM, HI, NM, TaT, KYa, YuT, $\mathrm{HK}$ and MM contributed to the acquisition of data. TS and YoT contributed to the analysis and interpretation of data, and drafted the manuscript. TS, YoT and KYo critically revised the manuscript. KYo supervised the study.

\section{Ethics approval and consent to participate}

Informed consent for participation in the study or use of their tissue was obtained from the patients.

\section{Patient consent for publication}

Written informed consent was obtained from each patient for publication of this case report and accompanying images.

\section{Competing interests}

K. Yoshida has received grants, personal fees and nonfinancial support from Chugai Pharmaceutical Co., Ltd. during the conduction of the study; grants and personal fees from Taiho Pharmaceutical Co., Ltd., Pfizer Inc., and Yakult Honsha Co., Ltd.; grants from Bristol-Myers Squibb; grants from Kyowa Hakko Kirin Co., Ltd. outside the submitted work; honoraria from Taiho Pharmaceutical Co., Ltd., Pfizer Inc., Chugai Pharmaceutical Co., Ltd., Kyowa Hakko Kirin Co., Ltd., and Yakult Honsha Co., Ltd.; and had a consultant or advisory relationship with Taiho Pharmaceutical Co., Ltd. and La Roche, Ltd. T. Takahashi has received honoraria for lectures from Takeda Pharmaceutical Co., Ltd. All remaining authors declare that they have no competing interests.

\section{References}

1. Merigliano S, Molena D, Ruol A, Zaninotto G, Cagol M, Scappin S and Ancona E: Chylothorax complicating esophagectomy for cancer: A plea for early thoracic duct ligation. J Thorac Cardiovasc Surg 119: 453-457, 2000.

2. Merrigan BA, Winter DC and O'Sullivan GC: Chylothorax. Br J Surg 84: 15-20, 1997.

3. Lagarde SM, Omloo JM, de Jong K, Busch OR, Obertop H and van Lanschot JJ: Incidence and management of chyle leakage after esophagectomy. Ann Thorac Surg 80: 449-454, 2005.

4. Miao L, Zhang Y, Hu H, Ma L, Shun Y, Xiang J and Chen H: Incidence and management of chylothorax after esophagectomy. Thorac Cancer 6: 354-358, 2015.
5. Itkin M, Kucharczuk JC, Kwak A, Trerotola SO and Kaiser LR: Nonoperative thoracicductembolization for traumatic thoracicduct leak: Experience in 109 patients. J Thorac Cardiovasc Surg 139: 584-589, 2010.

6. Nadolski GJ and Itkin M: Feasibility of ultrasound-guided intranodal lymphangiogram for thoracic duct embolization. J Vasc Interv Radiol 23: 613-616, 2012.

7. Yamamoto M, Miyata H, Yamasaki M, Maeda N, Miyazaki Y, Takahashi T, Kurokawa Y, Nakajima K, Takiguchi S, Mori M and Doki Y: Chylothorax after esophagectomy cured by intranodal lymphangiography: A case report. Anticancer Res 35: 891-895, 2015.

8. Ohkura Y, Ueno M, Shindoh J, Iizuka T, Ka H and Udagawa H: Risk factors for postoperative chylothorax after radical subtotal esophagectomy. Ann Surg Oncol 25: 2739-2746, 2018.

9. Loukas M, Wartmann CT, Louis RG Jr, Tubbs RS, Salter EG, Gupta AA and Curry B: Cisterna chyli: A detailed anatomic investigation. Clin Anat 20: 683-688, 2007.

10. The Japan Esophageal Society: Guidelines for Diagnosis and Treatment of Carcinoma of the Esophagus. Kanehara Co Ltd., Tokyo, 2017.

11. Hara H, Tahara M, Daiko H, Kato K, Igaki H, Kadowaki S, Tanaka Y, Hamamoto Y, Matsushita H, Nagase M and Hosoya Y: Phase II feasibility study of preoperative chemotherapy with docetaxel, cisplatin, and fluorouracil for esophageal squamous cell carcinoma. Cancer Sci 104: 1455-1460, 2013.

12. Watanabe M, Baba Y, Yoshida N, Ishimoto T, Nagai Y, Iwatsuki M, Iwagami $\mathrm{S}$ and Baba $\mathrm{H}$ : Outcomes of preoperative chemotherapy with docetaxel, cisplatin, and 5-fluorouracil followed by esophagectomy in patients with resectable node-positive esophageal cancer. Ann Surg Oncol 21: 2838-2844, 2014.

13. Yamashita K, Hosoda K, Moriya H, Katada C, Sugawara M, Mieno H, Komori S, Katada N and Watanabe M: Prognostic advantage of docetaxel/cisplatin/5-fluorouracil neoadjuvant chemotherapy in clinical stage II/III esophageal squamous cell carcinoma due to excellent control of preoperative disease and postoperative lymph node recurrence. Oncology 92: 221-228, 2017.

14. Tanaka Y, Yoshida K, Yamada A, Tanahashi T, Okumura N, Matsuhashi N, Yamaguchi K and Miyazaki T: Phase II trial of biweekly docetaxel, cisplatin, and 5-fluorouracil chemotherapy for advanced esophageal squamous cell carcinoma. Cancer Chemother Pharmacol 77: 1143-1152, 2016.

15. Staats BA, Ellefson RD, Budahn LL, Dines DE, Prakash UB and Offord $\mathrm{K}$ : The lipoprotein profile of chylous and nonchylous pleural effusions. Mayo Clin Proc 55: 700-704, 1980.

16. Lai FC, Chen L, Tu YR, Lin M and Li X: Prevention of chylothorax complicating extensive esophageal resection by mass ligation of thoracic duct: A random control study. Ann Thorac Surg 91: 1770-1774, 2011.

17. Fujita T and Daiko H: Efficacy and predictor of octreotide treatment for postoperative chylothorax after thoracic esophagectomy. World J Surg 38: 2039-2045, 2014.

18. Cerfolio RJ, Allen MS, Deschamps C, Trastek VF and Pairolero PC: Postoperative chylothorax. J Thorac Cardiovasc Surg 112: 1361-1365, 1996.

19. Kranzfelder M, Gertler R, Hapfelmeier A, Friess H and Feith M: Chylothorax after esophagectomy for cancer: Impact of the surgical approach and neoadjuvant treatment: Systematic review and institutional analysis. Surg Endosc 27: 3530-3538, 2013. 